\title{
Comparison of the tuberculin skin test and the QuantiFERON- TB Gold test in detecting latent tuberculosis in health care workers in Iran
}

\author{
Ehsan Mostafavi ${ }^{1,2 *}$, Mahshid Nasehi ${ }^{3,4 *}$, Abdolrazagh Hashemi Shahraki ${ }^{1,2}$, Saber Esmaeili ${ }^{1,2,5}$, \\ Ebrahim Ghaderi ${ }^{6,7}$, Saeed Sharafi ${ }^{3}$, Amin Doosti-Irani $^{1,8}$ \\ ${ }^{1}$ Department of Epidemiology, Pasteur Institute of Iran, Tehran; ${ }^{2}$ Research Centre for Emerging and Reemerging Infectious Diseases, Pasteur \\ Institute of Iran, Akanlu, Kabudar-Ahang, Hamadan; ${ }^{3}$ Center for Diseases Control and Prevention, Ministry of Health and Medical Education, \\ Tehran; ' ${ }^{4}$ Department of Epidemiology and Biostatistics, School of Public Health, Iran University of Medical Sciences, Tehran; ${ }^{5}$ Department of \\ Bacteriology, Faculty of Medical Sciences, Tarbiat Modares University, Tehran; ${ }^{6}$ Social Determinants of Health Research Center, Kurdistan \\ University of Medical Sciences, Sanandaj; ' 7 Department of Epidemiology and Biostatistics, School of Medicine, Kurdistan University of Medical \\ Sciences, Sanandaj; ${ }^{8}$ Department of Epidemiology and Biostatistics, School of Public Health, Tehran University of Medical Sciences, Tehran, Iran
}

OBJECTIVES: The tuberculin skin test (TST) and the QuantiFERON-TB Gold test (QFT) are used to identify latent tuberculosis infections (LTBIs). The aim of this study was to determine the agreement between these two tests among health care workers in Iran.

METHODS: This cross-sectional study included 177 tuberculosis (TB) laboratory staff and 67 non-TB staff. TST indurations of $10 \mathrm{~mm}$ or more were considered positive. The Student's t-test and the chi-square test were used to compare the mean score and proportion of variables between the TB laboratory staff and the non-TB laboratory staff. Kappa statistics were used to evaluate the agreement between these tests, and logistic regression was used to assess the risk factors associated with positive results for each test.

RESULTS: The prevalence of LTBIs according to both the QFT and the TST was 17\% (95\% confidence interval $[\mathrm{CI}], 12 \%$ to $21 \%$ ) and $16 \%$ (95\% CI, $11 \%$ to $21 \%$ ), respectively. The agreement between the QFT and the TST was $77.46 \%$, with a kappa of 0.19 (95\% CI, 0.04 to 0.34$)$.

CONCLUSIONS: Although the prevalence of LTBI based on the QFT and the TST was not significantly different, the kappa statistic was low between these two tests for the detection of LTBIs.

KEY WORDS: Latent tuberculosis, Tuberculin skin test, QuantiFERON-TB Gold, Health care workers

\section{INTRODUCTION}

It is estimated that one-third of the world's population currently has a latent tuberculosis infection (LTBI) as a result of in-

\section{Correspondence: Amin Doosti-Irani}

Department of Epidemiology, Pasteur Institute of Iran, 12 Farwardin Ave., Tehran 1316943551, Iran

Tel/Fax: +98-2166496448, E-mail: a_doostiirani@yahoo.com

${ }^{*}$ Mostafavi and Nasehi have contributed equally to this work as joint first authors.

Received: Jul 8, 2016, Accepted: Jul 24, 2016, Published: Jul 24, 2016

This article is available from: http://e-epih.org/

(C) 2016, Korean Society of Epidemiology

(C) This is an open-access article distributed under the terms of the Creative Commons Attribution License (http://creativecommons.org/licenses/by/4.0/), which permits unrestricted use, distribution, and reproduction in any medium, provided the original work is properly cited. fection with Mycobacterium tuberculosis [1]. It is estimated that approximately $10 \%$ of individuals with an LTBI may develop active tuberculosis (TB) during their lifetime [2]. The timely detection of LTBIs is important to prevent the development of active TB [3].

The tuberculin skin test (TST) is the most commonly used test to identify LTBIs. Although the TST is inexpensive and simple, facilitating its use, especially in developing countries, the validity and reliability of the TST is affected by many factors, including Bacillus Calmette-Guérin (BCG) vaccination, infection with non-TB Mycobacterium (NTM) species, the method of TST administration, the interpretation of the reaction, insufficient dosage, and cutaneous anergy [4-7].

In recent years, the QuantiFERON-TB Gold test (QFT), which is one of the newly developed interferon-gamma release assays 
(IGRAs) used to diagnose TB infections, has been used extensively for the detection of latent TB [8]. The test identifies the level of interferon (IFN)-gamma produced in reaction to $M$. tuberculosis-specific antigens [9]. IGRAs have several important advantages over the TST. The most important advantage of IGRAs is that, unlike TST, they are not influenced by factors such as BCG vaccination, most NTM species, or antigen dose $[8,10]$. Nevertheless, IGRAs have some important disadvantages, such as involving additional material costs and requiring a well-equipped laboratory and blood sampling with subsequent special handling to preserve the viability of lymphocytes [8]. However, IGRAs have a high specificity and a similar sensitivity to the TST for the detection of LTBIs [10,11].

Studies have shown poor agreement between IGRAs and the TST [12-14]. However, in countries with a high incidence of TB, an acceptable level of agreement has been reported between the TST and IGRA. In contrast, studies conducted in countries with an annual incidence of TB of $\leq 20$ per 100,000 have found poor agreement between the QFT and the TST [15]. More evidence is therefore needed to evaluate discrepancies in the results between IGRAs and theTST [10].

Given the lack of evidence regarding instances of disagreement between the TST and IGRAs in the detection of LTBIs among health care workers (HCWs) in Iran, the aim of this study was to determine the agreement between these two tests among Iranian HCWs.

\section{MATERIALS AND METHODS}

A proposal for this study was approved by the scientific committee of the Pasteur Institute of Iran. This cross-sectional study was performed between November 2013 and January 2014 in eight universities of the medical sciences, including Shiraz, Golestan, Shahid Beheshti, Iran, Tehran, Tabriz, Kermanshah, and Isfahan. All of these universities contain regional reference TB laboratories performing microscopic examinations, cultures, and drug sensitivity testing on $M$. tuberculosis isolates from throughout Iran.

In this study, all TB laboratory staff (177 participants) and a random sample of non-TB staff (67 participants), including administrative, finance, and service staff were included. Pregnant women were excluded from this study. A questionnaire was used to gather information regarding demographic variables, place and history of work, history of contact with TB patients, the presence of a BCG vaccination scar, and history of purified protein derivative skin tests.

Trained staff injected $0.1 \mathrm{~mL}$ ( 5 tuberculin units) of human tuberculin (Razi Vaccine and Serum Research Institute, Karaj, Iran) intradermally into the dorsal or volar surface of the fore- arm. TheTST response after 48 to 72 hours was read by a trained technician. TST reactions of $\geq 10 \mathrm{~mm}$ were considered positive, while reactions of $<10 \mathrm{~mm}$ were interpreted as negative [6].

The QFT was performed using a blood sample. Blood samples were obtained before the TST. At least $3 \mathrm{~mL}$ of blood was obtained from each participant, and then a 1-mL blood sample was transferred into each of three QuantiFERON-TB Gold tubes (Cellestis Ltd., Victoria, Australia). Blood samples with test antigens were incubated for 16 to 24 hours based on the kit instructions. The plasma samples were harvested into new labeled tubes and were delivered to the Pasteur Institute of Iran through a $4^{\circ} \mathrm{C}$ cold chain. The concentration of IFN-gamma (IU/ $\mathrm{mL}$ ) was measured using an automated enzyme-linked immunosorbent assay. The results of the test were interpreted using software supplied by the manufacturer (Cellestis Ltd.) including a cut-off point for the detection of IFN-gamma.

The individuals who had positive TST or QFT results were visited by an expert specialist physician. Active TB was diagnosed based on clinical findings, such as general symptoms (including fatigue, malaise, fever, weight loss, and anorexia) and chronic, productive cough with purulent sputum, in combination with a chest X-ray with radiological features consistent with TB disease.

The prevalence of LTBIs was estimated with $95 \%$ confidence intervals (CIs) using the QFT andTST.The concordance between these two tests was evaluated using proportion agreement and the kappa $(\kappa)$ statistic. The $\kappa$ statistic was interpreted as follows: $\kappa>0.75$ was considered to indicate excellent agreement, $\kappa<0.40$ to indicate poor agreement, and $\kappa$ between 0.40 and 0.75 to indicate fair to good agreement [16].

Both the Student's t-test and the chi-square test were used to compare means and proportions of variables between the TB laboratory staff and the non-TB laboratory staff. Logistic regression was used to identify risk factors associated with positive results for each test. Unadjusted and adjusted odds ratios (ORs) were reported to assess the effects of covariates on LTBI incidence. All statistical analyses were conducted using Stata version 11.0 (Stata Corp., College Station, TX, USA) and the results were reported with $95 \%$ CIs.

\section{RESULTS}

In this study, 244 participants, including 177 TB laboratory staff and 67 non-TB staff, were assessed for LTBIs using both the TST and QFT.The mean age of the TB lab staff and the nonTB staff was 36.75 years (standard error [SE], 0.54) and 39.40 years (SE, 1.15), respectively. Males comprised $60.25 \%$ (147 of 244) of the participants (Table 1 ).

The total estimated prevalence of LTBIs based on the TST 
Table 1. Demographic characteristics of the participants

\begin{tabular}{|c|c|c|c|c|c|c|}
\hline & \multicolumn{2}{|c|}{ TB lab staff } & \multicolumn{2}{|c|}{ Non-TB staff } & \multirow{2}{*}{$p$-value } & \multirow{2}{*}{ Total } \\
\hline & $n$ & $\%$ & $n$ & $\%$ & & \\
\hline \multicolumn{7}{|l|}{ Sex } \\
\hline Male & 100 & 56.50 & 47 & 70.15 & \multirow{2}{*}{0.05} & 147 \\
\hline Female & 77 & 43.50 & 20 & 29.85 & & 97 \\
\hline \multicolumn{7}{|l|}{ Age (yr) } \\
\hline $20-29$ & 29 & 15.85 & 10 & 14.93 & \multirow[t]{4}{*}{0.31} & 39 \\
\hline $30-39$ & 86 & 46.99 & 26 & 38.81 & & 112 \\
\hline $40-49$ & 56 & 30.60 & 22 & 32.84 & & 78 \\
\hline$\geq 50$ & 12 & 6.56 & 9 & 123.43 & & 21 \\
\hline \multicolumn{7}{|l|}{ Education level } \\
\hline No high school diploma & 17 & 9.60 & 12 & 17.91 & \multirow[t]{5}{*}{0.001} & 29 \\
\hline High school diploma & 13 & 7.34 & 30 & 44.78 & & 43 \\
\hline Associate degree & 59 & 33.33 & 8 & 11.94 & & 67 \\
\hline $\mathrm{BS}$ & 74 & 41.81 & 16 & 23.88 & & 90 \\
\hline Master of science and above & 14 & 7.91 & 1 & 1.49 & & 15 \\
\hline \multicolumn{7}{|l|}{ Job } \\
\hline Lab position requiring a BS & 85 & 48.02 & - & - & \multirow[t]{6}{*}{0.001} & 85 \\
\hline Technician & 60 & 33.90 & - & - & & 60 \\
\hline Service personnel in lab & 32 & 18.08 & - & - & & 32 \\
\hline Administrative staff & - & - & 42 & 62.69 & & 42 \\
\hline Finance staff & - & - & 10 & 14.93 & & 10 \\
\hline Service personnel & - & - & 15 & 22.39 & & 15 \\
\hline \multicolumn{7}{|l|}{ History of work (yr) } \\
\hline $0-4$ & 62 & 35.03 & 14 & 20.90 & \multirow[t]{5}{*}{0.001} & 76 \\
\hline $5-9$ & 42 & 23.73 & 10 & 14.93 & & 52 \\
\hline $10-14$ & 36 & 20.34 & 12 & 17.91 & & 48 \\
\hline $15-19$ & 25 & 14.12 & 13 & 19.40 & & 38 \\
\hline$\geq 20$ & 12 & 6.78 & 18 & 26.87 & & 30 \\
\hline History of BCG vaccination & 159 & 89.83 & 50 & 74.63 & & 209 \\
\hline
\end{tabular}

TB, tuberculosis; BS, bachelor of science; BCG, Bacillus Calmette-Guérin.

was $16 \%$ (95\% CI, $11 \%$ to $21 \%$ ). The prevalence of LTBIs based on the TST among males was significantly higher than among females $(p<0.05)$. Additionally, the prevalence of LTBIs based on this test increased with age, as subjects $\geq 50$ years old had a higher prevalence of LTBIs $(\mathrm{p}<0.05)$ (Table 2).

Logistic regression analysis showed the adjusted OR for TB lab staff in comparison to non-TB staff to be 0.31 (95\% CI, 0.11 to 0.93$)$. The adjusted OR for participants who had a history of contact with TB patients was 1.70 (95\% CI, 0.71 to 4.05), and positive TST results among participants with a history of BCG vaccination were more common than among those with no such history (OR, 2.23; 95\% CI, 0.67 to 7.43) (Table 3).

The difference in the prevalence of LTBIs measured using the QFT was not statistically significant across any subgroups. The total estimated prevalence of LTBIs based on the QFT was 17\% (95\% CI, $12 \%$ to $21 \%$ ). The prevalence of LTBIs among TB lab staff and non-TB staff was $19 \%$ (95\% CI, $12 \%$ to $24 \%$ ) and $13 \%$ (95\% CI, $5 \%$ to $23 \%$ ), respectively (Table 2 ).

According to the adjusted logistic regression, subjects with a history of work of 20 years or more were more likely to have positive QFT results than subjects with a history of work of zero to four years (OR, 2.64; 95\% CI, 0.53 to 13.09). Partici- pants with a history of BCG vaccination were less likely to be QFT-positive than others (OR, 0.50; 95\% CI, 0.21 to 1.23) (Table 3).

The overall agreement and $\kappa$ statistic were $77.46 \%$ and 0.19 (95\% CI, 0.04 to 0.34), respectively. Both theTST and QFT were positive in 13 and negative in 176 subjects (Table 4).

\section{DISCUSSION}

The results of this study showed that the estimated prevalence of LTBIs according to the QFT and the TST was $17 \%$ and $16 \%$, respectively. The estimated value of agreement between the QFT and the TST was $77.46 \%$, and the $\kappa$ statistic was 0.19 (95\% CI, 0.04 to 0.34 ).

The logistic regression analysis indicated different adjusted ORs associated with the QFT and the TST. The adjusted OR for people with $\geq 20$ years of work history was 2.64 times the baseline for the QFT and 0.54 times the baseline for the TST. These results were, however, not statistically significant due to the low sample size of this subgroup.

Another study among Iranian HCWs reported agreement and 
Table 2. Comparison of the prevalence of LTBIs according to the QFT and TST

\begin{tabular}{|c|c|c|c|c|c|c|c|}
\hline \multirow{2}{*}{ Variable } & \multicolumn{2}{|c|}{ QFT } & \multirow{2}{*}{ p-value } & \multicolumn{2}{|c|}{ TST } & \multirow{2}{*}{ p-value } & \multirow{2}{*}{$\mathrm{p}$-value } \\
\hline & Prevalence & $95 \% \mathrm{Cl}$ & & Prevalence & $95 \% \mathrm{Cl}$ & & \\
\hline \multicolumn{8}{|l|}{ Sex } \\
\hline Male & 0.17 & $0.11,0.23$ & \multirow[t]{2}{*}{0.92} & 0.20 & $0.13,0.26$ & \multirow[t]{2}{*}{$<0.05$} & 0.46 \\
\hline Female & 0.18 & $0.10,0.25$ & & 0.10 & $0.04,0.16$ & & 0.22 \\
\hline \multicolumn{8}{|l|}{ Age (yr) } \\
\hline $20-29$ & 0.10 & $0.03,0.24$ & \multirow[t]{4}{*}{0.13} & 0.03 & $0.00,0.13$ & \multirow[t]{4}{*}{$<0.05$} & 0.16 \\
\hline $30-39$ & 0.17 & $0.10,0.25$ & & 0.16 & $0.10,0.25$ & & 0.86 \\
\hline $40-49$ & 0.23 & $0.14,0.34$ & & 0.22 & $0.13,0.33$ & & 0.85 \\
\hline$\geq 50$ & 0.05 & $0.00,0.24$ & & 0.23 & $0.08,0.47$ & & 0.07 \\
\hline \multicolumn{8}{|l|}{ Education level } \\
\hline No high school diploma & 0.21 & $0.06,0.36$ & \multirow[t]{5}{*}{0.68} & 0.28 & $0.11,0.44$ & \multirow[t]{5}{*}{0.49} & 0.54 \\
\hline High school diploma & 0.09 & $0.00,0.18$ & & 0.12 & $0.02,0.21$ & & 0.50 \\
\hline Associate degree & 0.18 & $0.09,0.27$ & & 0.16 & $0.07,0.25$ & & 0.82 \\
\hline $\mathrm{BS}$ & 0.18 & $0.10,0.26$ & & 0.13 & $0.06,0.20$ & & 0.54 \\
\hline Master of science and above & 0.27 & $0.03,0.50$ & & 0.20 & $0.01,0.41$ & & 0.67 \\
\hline \multicolumn{8}{|l|}{ Job group } \\
\hline TB lab staff & 0.19 & $0.12,0.24$ & \multirow[t]{2}{*}{0.34} & 0.14 & $0.09,0.19$ & \multirow[t]{2}{*}{0.20} & 0.25 \\
\hline Non-TB staff & 0.12 & $0.05,0.22$ & & 0.21 & $0.11,0.31$ & & 0.39 \\
\hline \multicolumn{8}{|l|}{ Job } \\
\hline Lab position requiring a BS & 0.19 & $0.10,0.27$ & \multirow[t]{6}{*}{0.78} & 0.14 & $0.07,0.22$ & \multirow[t]{6}{*}{0.58} & 0.54 \\
\hline Technician & 0.17 & $0.07,0.26$ & & 0.17 & $0.07,0.26$ & & 0.81 \\
\hline Service personnel in lab & 0.22 & $0.07,0.37$ & & 0.09 & $0.01,0.20$ & & 0.17 \\
\hline Administrative staff & 0.14 & $0.04,0.25$ & & 0.19 & $0.07,0.31$ & & 0.56 \\
\hline Finance staff & - & - & & 0.10 & $0.09,0.30$ & & 0.31 \\
\hline Service personnel & 0.20 & $0.01,0.41$ & & 0.33 & $0.09,0.58$ & & 0.41 \\
\hline \multicolumn{8}{|l|}{ History of work (yr) } \\
\hline $0-4$ & 0.16 & $0.07,0.24$ & \multirow[t]{5}{*}{0.42} & 0.11 & $0.03,0.18$ & \multirow[t]{5}{*}{0.46} & 0.34 \\
\hline $5-9$ & 0.15 & $0.05,0.24$ & & 0.13 & $0.04,0.22$ & & 0.78 \\
\hline $10-14$ & 0.19 & $0.07,0.30$ & & 0.21 & $0.09,0.33$ & & 0.62 \\
\hline $15-19$ & 0.16 & $0.04,0.28$ & & 0.18 & $0.06,0.31$ & & 0.56 \\
\hline$\geq 20$ & 0.23 & $0.08,0.38$ & & 0.22 & $0.08,0.39$ & & 1.00 \\
\hline \multicolumn{8}{|l|}{$B C G$ vaccination } \\
\hline No & 0.26 & $0.11,0.40$ & \multirow[t]{3}{*}{0.15} & 0.11 & $0.01,0.22$ & \multirow[t]{2}{*}{0.42} & 0.12 \\
\hline Yes & 0.16 & $0.11,0.21$ & & 0.17 & $0.12,0.22$ & & 0.79 \\
\hline Total & 0.17 & $0.12,0.21$ & & 0.16 & $0.11,0.21$ & & 0.90 \\
\hline
\end{tabular}

LBTI, latent tuberculosis infection; TST, tuberculin skin test, QFT, QuantiFERON-TB Gold test; Cl, confidence interval; TB, tuberculosis; BS, bachelor of science; BCG, Bacillus Calmette-Guérin.

${ }^{1}$ For the comparison of the TST and QFT in each subgroup.

a $\kappa$ statistic of $73.8 \%$ and 0.39 between the tests, respectively [17]. The agreement between the two tests (TST and QFT) was very similar in that study and our study, but the $\kappa$ statistic was higher in their study. This discrepancy may reflect differences in the study population. The prevalence of TB in different regions of Iran is not the same [18-21], and the $\kappa$ statistic is influenced by the prevalence of a disease [22]. In the previous study [17], all subjects were HCWs in a hyper-endemic region. Although the overall agreement between the two tests was good, it is important to note that the measures of agreement have an important limitation; namely, these measures do not take into account the possibility that agreement may occur by chance alone [16].

In another study among HCWs in France, the agreement between the QFT and the TST was weak, and the $\kappa$ value was 0.11
[23]. In a study of HCWs in Turkey, the overall agreement and $\kappa$ statistic were $63.1 \%$ and 0.22 , respectively [24]. The $\kappa$ statistic between the QFT and the TST was found to be 0.22 in South Korean HCWs [25]. Among HCWs in the US, the agreement and $\kappa$ value between the QFT and TST were $63.2 \%$ and 0.31 (95\% CI, 0.27 to 0.35 ), respectively [26]. The $\kappa$ value between the QFT and TST in these studies [17,23-26] is almost the same as was found in our study and indicates poor agreement between the QFT and the TST.

According to the results of a meta-analysis, the $\kappa$ statistic between the QFT and TST among HCWs in countries with a high burden of TB (0.38) was significantly more than has been observed in low-burden countries (0.21) [27]. Therefore, it might be concluded that the agreement of the QFT and TST in popu- 
Table 3. Results of the TST and QFT according to probable risk factors

\begin{tabular}{|c|c|c|c|c|c|c|c|c|}
\hline \multirow{2}{*}{ Variable } & \multicolumn{4}{|c|}{ QFT } & \multicolumn{4}{|c|}{ TST } \\
\hline & Unadjusted & $p$-value & Adjusted & $p$-value & Unadjusted & $p$-value & Adjusted & $p$-value \\
\hline \multicolumn{9}{|l|}{ Sex } \\
\hline Female & 1.00 & & 1.00 & & 1.00 & & 1.00 & \\
\hline Male & $0.96(0.49,1.90)$ & 0.89 & $0.98(0.46,2.07)$ & 0.94 & $2.14(0.99,4.61)$ & 0.05 & $1.75(0.75,4.08)$ & 0.20 \\
\hline Age & $1.01(0.97,1.05)$ & 0.69 & $0.99(0.92,1.06)$ & 0.77 & $1.07(1.02,1.11)$ & 0.003 & $1.07(1.00,1.15)$ & 0.05 \\
\hline \multicolumn{9}{|l|}{ Education Level } \\
\hline No high school diploma & 1.00 & & 1.00 & & 1.00 & & 1.00 & \\
\hline High school diploma & $0.39(0.10,1.54)$ & 0.15 & $0.43(0.10,1.87)$ & 0.26 & $0.35(0.10,1.19)$ & 0.09 & $0.40(0.10,1.58)$ & 0.19 \\
\hline Associate degree & $0.84(0.28,2.49)$ & 0.70 & $0.81(0.22,2.95)$ & 0.75 & $0.52(0.18,1.46)$ & 0.21 & $1.27(0.35,4.55)$ & 0.72 \\
\hline Bachelor of science & $0.83(0.29,2.36)$ & 0.69 & $0.79(0.22,2.80)$ & 0.71 & $0.40(0.15,1.12)$ & 0.8 & $1.02(0.28,3.70)$ & 0.98 \\
\hline Master of science and above & $1.40(0.33,5.97)$ & 0.66 & $1.19(0.23,6.10)$ & 0.84 & $0.66(0.15,2.95)$ & 0.58 & $1.75(0.30,10.16)$ & 0.53 \\
\hline \multicolumn{9}{|l|}{ Job group } \\
\hline Non-lab staff & 1.00 & & 1.00 & & 1.00 & & 1.00 & \\
\hline TB lab staff & $1.48(0.67,3.28)$ & 0.34 & $1.88(0.67,5.29)$ & 0.23 & $0.62(0.30,1.26)$ & 0.20 & $2.23(0.11,0.93)$ & 0.04 \\
\hline \multicolumn{9}{|l|}{ History of work (yr) } \\
\hline $0-4$ & 1.00 & & 1.00 & & 1.00 & & 1.00 & \\
\hline $5-9$ & $0.97(0.37,2.57)$ & 0.95 & $1.00(0.36,2.74)$ & 0.99 & $1.32(0.45,3.90)$ & 0.61 & $1.07(0.35,3.30)$ & 0.9 \\
\hline $10-14$ & $1.23(0.47,3.18)$ & 0.67 & $1.42(0.49,4.13)$ & 0.52 & $2.24(0.81,6.14)$ & 0.12 & $1.24(0.41,3.75)$ & 0.71 \\
\hline $15-19$ & $1.00(0.34,2.90)$ & 1.00 & $1.30(0.36,4.61)$ & 0.69 & $1.92(0.64,5.76)$ & 0.25 & $0.77(0.21,2.85)$ & 0.69 \\
\hline$\geq 20$ & $1.62(0.57,4.62)$ & 0.36 & $2.64(0.53,13.09)$ & 0.24 & $2.58(0.84,7.92)$ & 0.09 & $0.54(0.11,2.68)$ & 0.45 \\
\hline \multicolumn{9}{|l|}{ Contact with TB patients } \\
\hline No & 1.00 & & 1.00 & & 1.00 & & 1.00 & \\
\hline Yes & $0.95(0.49,1.85)$ & 0.87 & $0.73(0.34,1.56)$ & 0.42 & $1.43(0.72,2.84)$ & 0.31 & $1.70(0.71,4.05)$ & 0.23 \\
\hline \multicolumn{9}{|l|}{ BCG vaccination } \\
\hline No & 1.00 & & 1.00 & & 1.00 & & 1.00 & \\
\hline Yes & $0.54(0.23,1.26)$ & 0.15 & $0.50(0.21,1.23)$ & 0.13 & $1.56(0.52,4.70)$ & 0.43 & $2.23(0.67,7.43)$ & 0.19 \\
\hline
\end{tabular}

Values are presented as odds ratio (95\% confidence interval).

TST, tuberculin skin test, QFT, QuantiFERON-TB Gold test; TB, tuberculosis; BCG, Bacillus Calmette-Guérin.

Table 4. Overall agreement and kappa values between the QFT and TST results

\begin{tabular}{|c|c|c|c|c|c|c|c|}
\hline \multirow{2}{*}{ Group } & \multirow{2}{*}{$\begin{array}{l}\text { TST results (cut-off } \\
\text { point of } \geq 10 \mathrm{~mm} \text { ) }\end{array}$} & \multicolumn{2}{|c|}{ QFT results } & \multirow{2}{*}{ Total } & \multirow{2}{*}{ Agreement (\%) } & \multirow{2}{*}{ Kappa (95\%Cl) } & \multirow{2}{*}{$\mathrm{p}$-value } \\
\hline & & Negative & Positive & & & & \\
\hline \multirow[t]{3}{*}{ TB staff } & Negative & 128 & 24 & 152 & 77.40 & $0.18(0.00,0.35)$ & 0.008 \\
\hline & Positive & 16 & 9 & 25 & & & \\
\hline & Total & 144 & 33 & 177 & & & \\
\hline \multirow[t]{3}{*}{ Non-TB staff } & Negative & 48 & 5 & 53 & 77.61 & $0.22(-0.06,0.50)$ & 0.03 \\
\hline & Positive & 10 & 4 & 14 & & & \\
\hline & Total & 58 & 9 & 67 & & & \\
\hline \multirow[t]{3}{*}{ BCG vaccinated } & Negative & 150 & 22 & 172 & 76.92 & $0.16(-0.00,0.32)$ & 0.01 \\
\hline & Positive & 26 & 10 & 36 & & & \\
\hline & Total & 176 & 32 & 208 & & & \\
\hline \multirow[t]{3}{*}{ Non-BCG vaccinated } & Negative & 25 & 7 & 32 & 75.00 & $0.18(-0.16,0.53)$ & 0.11 \\
\hline & Positive & 2 & 2 & 4 & & & \\
\hline & Total & 27 & 9 & 36 & & & \\
\hline \multirow[t]{3}{*}{ All participants } & Negative & 176 & 29 & 205 & 77.46 & $0.19(0.04,0.34)$ & 0.001 \\
\hline & Positive & 26 & 13 & 39 & & & \\
\hline & Total & 202 & 33 & 244 & & & \\
\hline
\end{tabular}

QFT, QuantiFERON-TB Gold test; TST, tuberculin skin test; Cl, confidence interval; TB, tuberculosis; BCG, Bacillus Calmette-Guerin.

lations with a high risk of TB infection seems to be better than in low-risk populations. However, in the high-risk population investigated in the current study, the agreement was also found to be poor. The differences in the $\kappa$ statistic among various stud- ies may be due to differences in the prevalence of LTBIs in various study populations. Moreover, one of the limitations of the $\kappa$ statistic is its dependence on prevalence; namely, the $\kappa$ statistic is higher in populations in which the prevalence is high than in 
populations with a lower prevalence [16].

One study [17] showed that a family history of TB was a potential risk factor for LTBI based on the QFT (OR,7.96) and TST (OR, 4.91). We did not observe any analogous findings. In our study, the adjusted OR for contact with TB patients was 0.73 (95\% CI, 0.34 to 1.56 ) for the QFT and 1.70 (95\% CI, 0.71 to 4.05$)$ for the TST. One reason for this may be the availability and use of suitable protection while working with patients and/or their sputum samples. In the patients' family members, contact was not found to be protective.

BCG vaccination had a non-significant protective association with LTBIs based on the QFT (OR, 0.50; 95\% CI, 0.21 to 1.23), while the association of BCG vaccination with LTBI based on the TST was not significant (OR, 2.23; 95\% CI, 0.67 to 7.43). This inconsistency may be due to the influence of BCG vaccination on the results of the TST, since other studies have shown that the results of the TST were influenced by BCG vaccination $[8,10]$. In a study among HCWs in the US, a positive TST with a negative QFT was associated with BCG vaccination (OR, 25.1; $95 \%$ CI, 15.5 to 40.5 ) [26].

This study was conducted in eight universities of medical science in Iran. All QFT samples were sent to the Department of Epidemiology of the Pasteur Institute of Iran, and the tests were performed by a trained lab expert. The TST tests were conducted by different lab experts and technicians in the eight universities. Although we trained all lab experts and technicians in the method of TST testing, heterogeneity may exist in TST testing by multiple lab experts and technicians, so this factor may have affected the results of the TST test.

The prevalence of LTBIs according to both the QFT and TST was considerable. Although the prevalence of LTBIs based on the QFT and TST was not significantly different, and the overall agreement between both tests was good, the $\kappa$ statistic was low between these two tests. The $\kappa$ value among the non-BCG vaccinated group was higher than among the vaccinated group. It seems the use of the QFT test, due to its high cost, is only appropriate in cases where the results of a TST could be affected by BCG vaccination or NTM species.

\section{ACKNOWLEDGEMENTS}

We would like to thank all TB staff members who participated, as well as the staff of the TB office of the Center for Disease Control of the Ministry of Health and Medical Education. This study was supported by grants from the United Nations Development Programme (UNDP), no. PSC/13/10.

\section{CONFLICT OF INTEREST}

The authors have no conflicts of interest to declare for this study.

\section{ORCID}

Ehsan Mostafavi http://orcid.org/0000-0002-1997-517X

Mahshid Nasehi http://orcid.org/0000-0002-2913-4294

Abdolrazagh Hashemi Shahraki http://orcid.org/0000-00031852-194X

Saber Esmaeili http://orcid.org/0000-0003-1920-1737

Ebrahim Ghaderi http://orcid.org/0000-0002-2303-1657

Saeed Sharafi http://orcid.org/0000-0002-6875-3426

Amin Doosti-Irani http://orcid.org/0000-0003-0623-7503

\section{REFERENCES}

1. World Health Organization. Tuberculosis: fact sheet no. 104; 2012 [cited 2014 Nov 26]. Available from: http://www.who.int/mediacentre/factsheets/who104/en/print.html.

2. Comstock GW, Livesay VT, Woolpert SF. The prognosis of a positive tuberculin reaction in childhood and adolescence. Am J Epidemiol 1974;99:131-138.

3. Fitzgerald DW, Sterling TR, Has DW. Mycobacterium tuberculosis. In: Mandell GL, Douglas RG, Bennett JE, Dolin R, Blaser MJ, editors. Mandell, Douglas, and Bennett's principles and practice of infectious diseases. 7th ed. Philadelphia: Elsevier; 2010, p. 3129-3159.

4. Farhat M, Greenaway C, Pai M, Menzies D. False-positive tuberculin skin tests: what is the absolute effect of BCG and non-tuberculous mycobacteria? Int J Tuberc Lung Dis 2006;10:1192-1204.

5. Lee E, Holzman RS. Evolution and current use of the tuberculin test. Clin Infect Dis 2002;34:365-370

6. Huebner RE, Schein MF, Bass JB Jr. The tuberculin skin test. Clin Infect Dis 1993;17:968-975.

7. Nayak S, Acharjya B. Mantoux test and its interpretation. Indian Dermatol Online J 2012;3:2-6.

8. Mazurek GH, Jereb J, Lobue P, Iademarco MF, Metchock B, Vernon A, et al. Guidelines for using the QuantiFERON-TB Gold test for detecting Mycobacterium tuberculosis infection, United States. MMWR Recomm Rep 2005;54:49-55.

9. Mazurek GH, Jereb J, Vernon A, LoBue P, Goldberg S, Castro K, et al. Updated guidelines for using interferon gamma release assays to detect Mycobacterium tuberculosis infection - United States, 2010. MMWR Recomm Rep 2010;59:1-25.

10. Menzies D, Pai M, Comstock G. Meta-analysis: new tests for the diagnosis of latent tuberculosis infection: areas of uncertainty and recommendations for research. Ann Intern Med 2007;146:340-354.

11. Pollock NR, Campos-Neto A, Kashino S, Napolitano D, Behar SM, Shin D, et al. Discordant QuantiFERON-TB Gold test results among US healthcare workers with increased risk of latent tuberculosis infection: a problem or solution? Infect Control Hosp Epidemiol 2008; 29:878-886.

12. Talati NJ, Seybold U, Humphrey B, Aina A, Tapia J, Weinfurter P, et al. Poor concordance between interferon-gamma release assays and 
tuberculin skin tests in diagnosis of latent tuberculosis infection among HIV-infected individuals. BMC Infect Dis 2009;9:15.

13. Chkhartishvili N, Kempker RR, Dvali N, Abashidze L, Sharavdze L, Gabunia P, et al. Poor agreement between interferon-gamma release assays and the tuberculin skin test among HIV-infected individuals in the country of Georgia. BMC Infect Dis 2013;13:513.

14. Connell TG, Ritz N, Paxton GA, Buttery JP, Curtis N, Ranganathan SC. A three-way comparison of tuberculin skin testing, QuantiFERON-TB gold and T-SPOT.TB in children. PLoS One 2008;3:e2624.

15. Swindells JE, Aliyu SH, Enoch DA, Abubakar I. Role of interferongamma release assays in healthcare workers. J Hosp Infect 2009;73: 101-108.

16. Szklo M, Nieto FJ. Epidemiology: beyond the basics. 3rd ed. Burlington: Jones \& Bartlett Learning; 2014, p. 338-340.

17. Hashemi Shahri M, Fallah Ghajary A, Ansari Moghaddam A, Khadem Sameni F, Fayyaz Jahani F, Ahmadnezhad E. To compare the performance of Quanti-FERON with the tuberculin skin test for identifying latent tuberculosis infection. Iran J Epidemiol 2012;7:57-65 (Persian).

18. Mirhaghani L, Nasehi M. National tuberculosis program in Iran. Tehran: Ministry of Health (Nashr-e Seda); 2002, p. 15-20 (Persian).

19. Khazaei HA, Rezaei N, Bagheri GR, Dankoub MA, Shahryari K, Tahai A, et al. Epidemiology of tuberculosis in the Southeastern Iran. Eur J Epidemiol 2005;20:879-883.

20. Rafiee S, Besharat S, Jabbari A, Golalipour F, Nasermoaadeli A. Epidemiology of tuberculosis in northeast of Iran: a population-based study. Iran J Med Sci 2009;34:193-197.

21. Sofian M, Zarinfar N, Mirzaee M, Nejad SA. Epidemiology of tuberculosis in Arak, Iran. Faslnamahi Kumish 2009;10:261-266 (Persian).

22. Sim J, Wright CC. The kappa statistic in reliability studies: use, interpretation, and sample size requirements. Phys Ther 2005;85:257-268.

23. Tripodi D, Brunet-Courtois B, Nael V, Audrain M, Chailleux E, Germaud $\mathrm{P}$, et al. Evaluation of the tuberculin skin test and the interferon-gamma release assay for TB screening in French healthcare workers. J Occup Med Toxicol 2009;4:30.

24. Ozdemir D, Annakkaya AN, Tarhan G, Sencan I, Cesur S, Balbay O, et al. Comparison of the tuberculin skin test and the quantiferon test for latent Mycobacterium tuberculosis infections in health care workers in Turkey. Jpn J Infect Dis 2007;60:102-105.

25. Jo KW, Hong Y, Park JS, Bae IG, Eom JS, Lee SR, et al. Prevalence of latent tuberculosis infection among health care workers in South Korea: a multicenter study. Tuberc Respir Dis (Seoul) 2013;75:1824.

26. Dorman SE, Belknap R, Graviss EA, Reves R, Schluger N, Weinfurter $\mathrm{P}$, et al. Interferon- $\gamma$ release assays and tuberculin skin testing for diagnosis of latent tuberculosis infection in healthcare workers in the United States. Am J Respir Crit Care Med 2014;189:77-87.

27. Doosti-Irani A, Ayubi E, Mostafavi E. Tuberculin and QuantiFERON-TB-Gold tests for latent tuberculosis: a meta-analysis. Occup Med (Lond) 2016;66:437-445. 